Check for updates

Cite this: RSC Adv., 2017, 7, 22913

Received 23rd February 2017 Accepted 10th April 2017

DOI: $10.1039 / c 7 r a 02253 k$

rsc.li/rsc-advances

\title{
A simple and sensitive sensor based on a molecularly imprinted polymer-modified carbon paste electrode for the determination of curcumin in foods
}

\author{
Q. Zhou, (DD *a H. Y. Zhai, ${ }^{a}$ Y. F. Pan*b and K. Li ${ }^{\text {a }}$
}

A highly sensitive and selective molecularly imprinted polymer-modified carbon paste electrode (MIP-CPE) was designed. The curcumin molecularly imprinted polymer (CUR-MIP) was prepared by thermally induced precipitation polymerization, using curcumin (CUR) as the template molecule, methacrylic acid (MAA) as the functional monomer, 2,2' -azodiisobutyronitrile (AIBN) as the initiator, and ethylene glycol dimethacrylate (EDGMA) as the cross-linker. The modified electrode was prepared by mixing the polymer, graphite powder, and paraffin oil in specific proportions. The electrochemical behaviour of CUR on this electrode was studied, and a method for direct CUR determination using this electrode was established. The results showed that CUR exhibited a distinct oxidation peak $\left(E_{\mathrm{pa}}\right)$ at $0.434 \mathrm{~V}$ ( $\mathrm{vs}$. SCE) in the mixed phosphate buffer solution (PBS) at $\mathrm{pH}$ 3.06. The optimum addition ratio of the polymer in the modified electrode was $20 \%$, and the peak current $\left(/_{\mathrm{pa}}\right)$ was 4.5 -fold higher than that of the bare CPE. The $I_{\mathrm{pa}}$ showed a good linear relationship with concentration in the range $0.1-50 \mu \mathrm{M}$, and the detection limit was $10.1 \mathrm{nM}$. The method was applied to determine CUR contents in food, giving recovery rates of 90.77-105.7\%.

\section{Introduction}

Curcumin (CUR, Scheme 1) is a diketone compound used widely in food and medicine. It is extracted from the roots of certain plants of the Zingiberaceae and Araceae families. It has been used as a natural pigment in the food industry due to its bright yellow colour, mainly in canned foods, intestinal products, cakes, beverages, and other dyeing applications. ${ }^{1}$ Pharmacologically, CUR, or its sodium salt, has antibacterial, antitumour, hypoglycaemic, and antioxidant effects..$^{2-7}$ In traditional Chinese medicine, CUR is believed to help blood circulation, warm the meridians, and cure pain caused by rheumatism and blood stasis.

In China, Japan, the European Union, and other regions, CUR can be legally added to food. However, in September 2010, the Food Additives and Nutritional Enhancers Science Committee of the European Food Safety Authority was asked to re-evaluate the safety of CUR as a food colouring. The acceptable daily intake amount may also be redefined. Therefore, the fast and convenient determination of CUR content in food has become necessary. Currently, CUR determination is mainly

${ }^{a}$ Department of Pharmacy, GuangDong Pharmaceutical University, GuangZhou, 510006, PR China. E-mail: kytxor@163.com; Fax: +86 2039352128; Tel: +86 02039352135

${ }^{b}$ Department of Chemistry, GuangDong Pharmaceutical University, ZhongShan, 528458, PR China. E-mail: p39352353@126.com performed by HPLC, HPTLC, LC-MS, TLCS, CE, and spectrophotometry. ${ }^{8-16}$ However, the pretreatment process for these methods is tedious, and sensitivity and selectivity vary. The electrochemical determination of CUR has rarely been reported. Afzalia et al. detected CUR in urine and turmeric samples using a ferrocene-nanofiber carbon paste electrode, which gave a linear calibration graph in the range $0.1-500 \mu \mathrm{M}$ curcumin with a detection limit of $24 \mathrm{nM} .{ }^{17}$ Cakir $e t$ al. used a palladium nanoparticle (PdNP)-coated graphite electrode to detect curcumin in samples of turmeric powder, which gave a linear calibration graph in the range $3.0 \times 10^{-8} \mathrm{M}$ to $6.0 \times 10^{-7} \mathrm{M}$ with a detection limit of $2.2 \times 10^{-8} \mathrm{M}^{18}$ Ziyatdinova et al. determined curcumin in spices using a glassy carbon electrode in $0.1 \mathrm{M} \mathrm{LiClO}_{4}$ in ethanol, which gave a linear calibration graph in the range $9.9 \times 10^{-6}$ to $1.07 \times 10^{-4} \mathrm{M}$ curcumin with a detection limit of $4.1 \times 10^{-6} \mathrm{M}^{19}$

Chemically modified carbon paste electrodes (CMCPEs) are composed of a modifier and carbon paste, which provides<smiles>COc1ccc(/C=C/C(=O)CC(=O)/C=C/c2ccc(OC)c(O)c2)cc1O</smiles>

Scheme 1 Chemical structure of curcumin. 
a specific function to the modified electrode. CMCPEs enhance the selectivity and sensitivity of the CPE, combining separation, enrichment, and selective determination. ${ }^{\mathbf{2 0 - 2 2}}$ Therefore CMCPEs have become popular in electrical analysis research. Our group has made progress in researching modified carbon paste electrodes. ${ }^{23-25}$ MIP has good stereospecific recognition and has been widely used in chromatographic separation, antibody or receptor simulation, biosensors, enzyme simulation, and catalytic synthesis, among other applications. In this paper, the electrochemical behaviour of CUR was studied using MIP-CPE as the working electrode. The direct determination of CUR using this electrode was established and applied to the determination of CUR in food, giving excellent results.

\section{Experimental}

\subsection{Apparatus and chemicals}

Cyclic voltammetry (CV) was performed using an Ingsens-1100 series handheld electrochemical workstation (Ingsens Instruments, Guangzhou, China). A conventional three-electrode system comprising MIP-CPE as the working electrode, SCE (Chenhua Instruments, Shanghai, China) as the reference electrode, and a platinum electrode (Chenhua Instruments, Shanghai, China) as the indicating electrode. A pH meter (pHS25, Leici Instrument, Shanghai, China) with a double junction glass electrode was used to determine the $\mathrm{pH}$ of solutions.

CUR $(\geq 98 \%)$ was purchased from the China Institute of Pharmaceutical and Biological Products, graphite powder (SP, $40 \mathrm{~nm}$ ) was obtained from the China National Pharmaceutical Group Chemical Reagent Co., Ltd. MAA (AR), EDGMA (AR), AIBN (AR), and liquid paraffin (HPLC) were purchased from Shanghai Aladdin Reagent Co., Ltd. Other chemicals were analytically pure, and deionized water was used in all experiments. Curcuma powder and cookies (Meixin West Bakery) were purchased from a supermarket in Guangzhou.

\subsection{Preparation of $\mathrm{MIP}^{26,27}$}

CUR (1 mM) was accurately weighed and placed in a roundbottom flask. $\mathrm{CH}_{3} \mathrm{OH}$ (120 mL), MAA (4 mM), EDGMA (20 $\mathrm{mM}$ ), and AIBN (30 mg) were then added and mixed well, followed by nitrogen purging for $10 \mathrm{~min}$. The flask was sealed and placed in a constant temperature water bath for polymerization at $60{ }^{\circ} \mathrm{C}$ for $24 \mathrm{~h}$. The product was rinsed with a mixed solution of methanol-acetic acid ( $9: 1)$ until no CUR was detected in the eluent. Methanol was used to remove the residual formic acid. The product was dried in the oven at $60{ }^{\circ} \mathrm{C}$ to obtain CUR-MIP.

A blank or a non-imprinted polymer (NIP) was prepared using the same method in the absence of template.

\subsection{Electrode fabrication}

Fine graphite powder, MIP, and paraffin oil were mixed in certain ratios and packed into a $2.0 \mathrm{~mm}$ radius glass tube. A copper wire was inserted from the back as the electrical contact. The electrode surface was polished using soft white paper, and the MIP-CPE was then prepared.
An NIP-CPE was prepared using the same method, but replacing MIP with NIP.

\subsection{Preparation of buffer solution and standard solution}

Mixed phosphate solid reagent $\left(\mathrm{KH}_{2} \mathrm{PO}_{4} 3.388 \mathrm{~g}+\mathrm{Na}_{2} \mathrm{HPO}_{4}\right.$ $3.533 \mathrm{~g}$ ) was dissolved in distilled water and transferred into a $250 \mathrm{~mL}$ volumetric flask to obtain PBS of $\mathrm{pH}$ 6.86. The $\mathrm{pH}$ was adjusted using $1 \mathrm{M} \mathrm{HCl}$ solution.

A CUR standard sample $(0.07369 \mathrm{~g})$ was accurately weighed and dissolved in absolute ethanol. The solution was transferred to a $200 \mathrm{~mL}$ brown volumetric flask. A $1.0 \mathrm{mM}$ CUR standard solution was obtained and stored in a $4{ }^{\circ} \mathrm{C}$ refrigerator. The solution was diluted to the desired concentration using PBS at $\mathrm{pH} 3.06$.

\subsection{Sample pretreatment}

Small samples of curcuma powder and cookies were ground and dried at $70{ }^{\circ} \mathrm{C}$. The curcuma powder $(0.2 \mathrm{~g})$ and curcuma cookie powder $(1 \mathrm{~g})$ were weighed and placed in separate conical flasks with stoppers. Next, 95\% ethanol $(20 \mathrm{~mL})$ was added to each flask for extraction under ultrasonication for $30 \mathrm{~min}$, followed by filtration. The sample residue was also extracted using this method. The filtrate was combined and transferred into a $100 \mathrm{~mL}$ volumetric flask to be stored at $4{ }^{\circ} \mathrm{C}$. For use, $2 \mathrm{~mL}$ of this solution was diluted to the desired concentration using PBS (18 mL, pH 3.06).

\subsection{Electrochemical measurement}

The solution was transferred into a small beaker with the threeelectrode system. The accumulation time $\left(t_{\text {acc }}\right)$ was set to $20 \mathrm{~s}$, and the electrode was scanned in the range $0-1.0 \mathrm{~V}$ at a scan rate of $50 \mathrm{mV} \mathrm{s}^{-1}$. The peak potential $\left(E_{\mathrm{pa}}\right)$ and peak current $\left(I_{\mathrm{pa}}\right)$ were recorded at $0.434 \mathrm{~V}$.

At the end of each measurement, the working electrode was placed in a $1 \mathrm{M} \mathrm{NaOH}$ solution and scanned for five cycles to remove any absorbed CUR.

The recovery study was conducted using a standard addition method. Different amounts of CUR were added to the sample solution, and the measured peak current was recorded using the electrode present.

\section{Results and discussion}

\subsection{Electrochemical behaviour of CUR}

Three different electrodes, MIP-CPE, NIP-CPE, and CPE, were used in a $10 \mu \mathrm{M}$ CUR solution for cyclic voltammetry scans, as described in Section 2.6. The results are shown in Fig. 2. The oxidation peak $\left(E_{\mathrm{pa}}\right)$ of CUR on the bare CPE was $0.434 \mathrm{~V}$, and the reduction peak $\left(E_{\mathrm{pc}}\right)$ was $0.338 \mathrm{~V}$ (Fig. 1c), which indicated that the oxidation-reduction reaction of CUR on CPE was quasireversible. The response of CUR on MIP-CPE was the highest (Fig. 1a). Compared with the bare CPE, the $I_{\text {pa }}$ increased approximately 4.5 -fold. This increase was probably due to the specific ability of MIP to recognize the template molecules. The CUR molecules could diffuse to the surface of the electrode through polymer pores and undergo a redox reaction. 


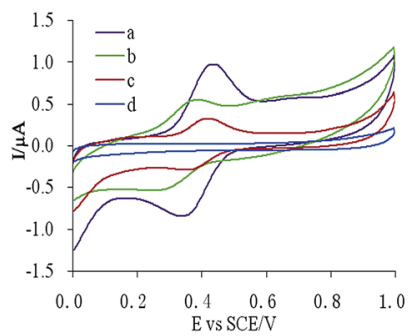

Fig. 1 Cyclic voltammetry of CUR on three different electrodes (a) MIP-CPE in $10 \mu \mathrm{M}$ CUR solution, (b) NIP-CPE in $10 \mu \mathrm{M}$ CUR solution, (c) bare CPE in $10 \mu \mathrm{M}$ CUR solution, and (d) bare CPE in PBS blank solution.

Meanwhile, due to the enrichment effect of MIP on the template molecules, the sensitivity of the electrode was increased, and the anti-interference ability of the electrode was improved.

\subsection{Optimization of measurement conditions}

3.2.1 Composition of polymer-modified CPE. Electrodes were prepared according to the method described in Section 2.3 , for which the ratios of graphite to MIP were $90: 10,80: 20$, $70: 30$, and $60: 40(\mathrm{~m} / \mathrm{m})$. The resulting electrodes were scanned in a $10 \mu \mathrm{M}$ CUR solution, and the results are shown in Fig. 2. Probably due to the functional group and pore of MIP having a specific recognition ability for the template molecule, adding MIP into the electrode material increased the adsorption capacity of the template molecule on the surface of the electrode, which increased the peak current. Higher peak currents and better peak shapes were obtained when the ratio of graphite to MIP was $80: 20$. Therefore, this optimized electrode composition was used to prepare modified electrodes.

3.2.2 Selection of buffer solution. Tests were performed in buffer solutions, such as $\mathrm{H}_{3} \mathrm{PO}_{4}-\mathrm{KH}_{2} \mathrm{PO}_{4}, \mathrm{NH}_{3}-\mathrm{NH}_{4} \mathrm{Cl}$, HAcNaAc, PBS, BR, $\mathrm{HCl}, \mathrm{Na}_{2} \mathrm{~B}_{4} \mathrm{O}_{7}$, and $\mathrm{KHC}_{4} \mathrm{H}_{4} \mathrm{O}_{6}$. The results showed that CUR had signals in both HAc-NaAc and $\mathrm{Na}_{2} \mathrm{~B}_{4} \mathrm{O}_{7}$ solution, but with a small peak current and poor stability. However, CUR showed an excellent peak shape, maximum peak current, and good stability in PBS solution. Therefore, PBS was selected as the buffer solution for subsequent tests.

3.2.3 Effect of solution $\mathrm{pH}$ on peak current and peak potential. Buffer solution $\mathrm{pH}$ had a significant effect on the cyclic voltammetry (CV) results. The curves of the linearly swept

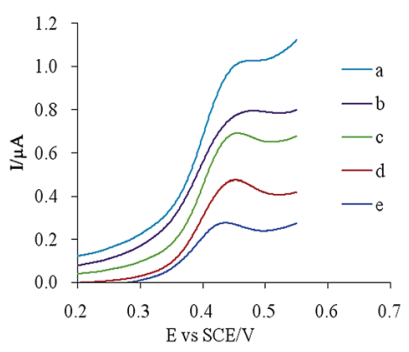

Fig. 2 Effect of MIP amount in the modified electrode at $10 \mu \mathrm{M}$ CUR peak current: (a) $80: 20$, (b) $90: 10$, (c) $70: 30$, (d) $60: 40$, and (e) bare CPE. voltammograms, peak current, and peak potential vs. $\mathrm{pH}$ are shown in Fig. 3A. The CUR peak current at different $\mathrm{pH}$ values showed no regularity (Fig. 3B), while the best peak shape and maximum peak current were observed at $\mathrm{pH}$ 3.06. Therefore, PBS solution at $\mathrm{pH} 3.06$ was used for subsequent tests.

CUR has a yellow or orange-yellow colour in acidic or neutral environments, and its peak potential decreases with increasing $\mathrm{pH}$. Over the $\mathrm{pH}$ range 2.54-7.52, the peak potential was a linear function of $\mathrm{pH}$ (Fig. 3C), following the equation $E=-0.054 \mathrm{pH}$ $+0.688, r=0.9990$, for which the slope was 0.054 and close to 0.059. According to the Nernst equation, the reaction of CUR on the electrode was a redox process with equal electrons and protons.

3.2.4 Effect of accumulation time on peak current. The effect of accumulation time $\left(t_{\mathrm{acc}}\right)$ on peak current was investigated at two different CUR concentrations. At $10 \mu \mathrm{M}$, the peak current reached a maximum at $15 \mathrm{~s}$, while at $1 \mu \mathrm{M}$, the peak current reached a maximum at $25 \mathrm{~s}$. This result indicated that lower-concentration CUR solutions required longer accumulation times. Considering peak shape, time, cost, and other factors, a $t_{\mathrm{acc}}$ of $20 \mathrm{~s}$ was used for subsequent tests.

\subsection{Reaction mechanism study}

The effect of scanning rate on the $10 \mu \mathrm{M}$ CUR peak current and peak potential was studied using cyclic voltammetry (Fig. 4A). The results showed that the peak current of CUR and $v$ exhibited a linear relationship in the scanning rate range $10-800 \mathrm{mV} \mathrm{s}^{-1}$ (Fig. 4B), with the equation $I_{\mathrm{pa}}=0.0078 v+0.0704, r=0.9998$. This result indicated that the oxidation of CUR was an absorption-controlled process. Additionally, the peak current increased gradually with increasing scanning rate. To obtain a better signal-to-noise ratio and peak shape, a scanning rate of $50 \mathrm{mV} \mathrm{s}^{-1}$ was used for subsequent experiments.

$E_{\text {pa }}$ and $\ln v$ also had a good linear relationship (Fig. 4C), with the following equation:
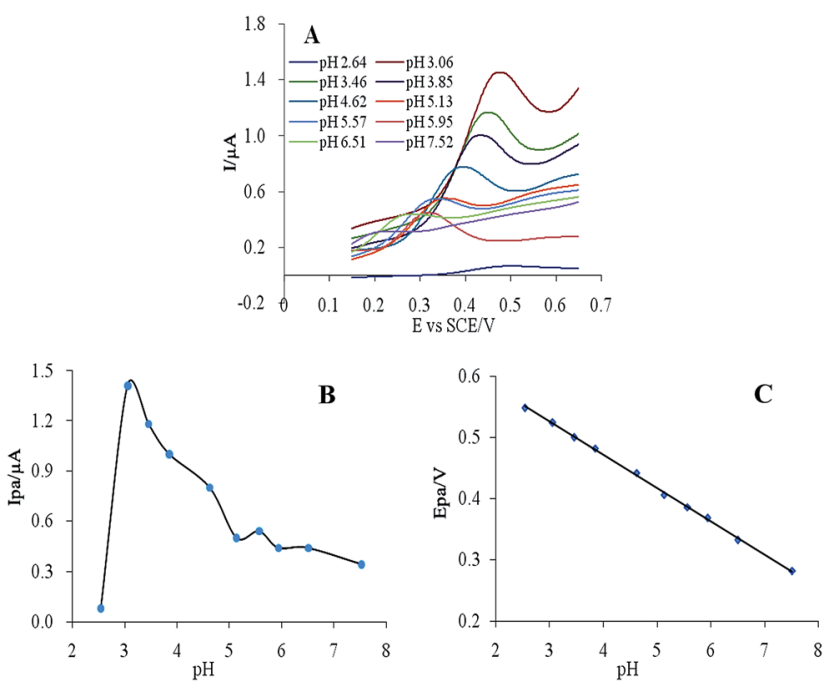

Fig. 3 (A) Cyclic voltammograms of $10 \mu \mathrm{M}$ CUR in PBS at pH 2.647.52. Effect of $\mathrm{pH}$ on the (B) peak current and (C) potential. 

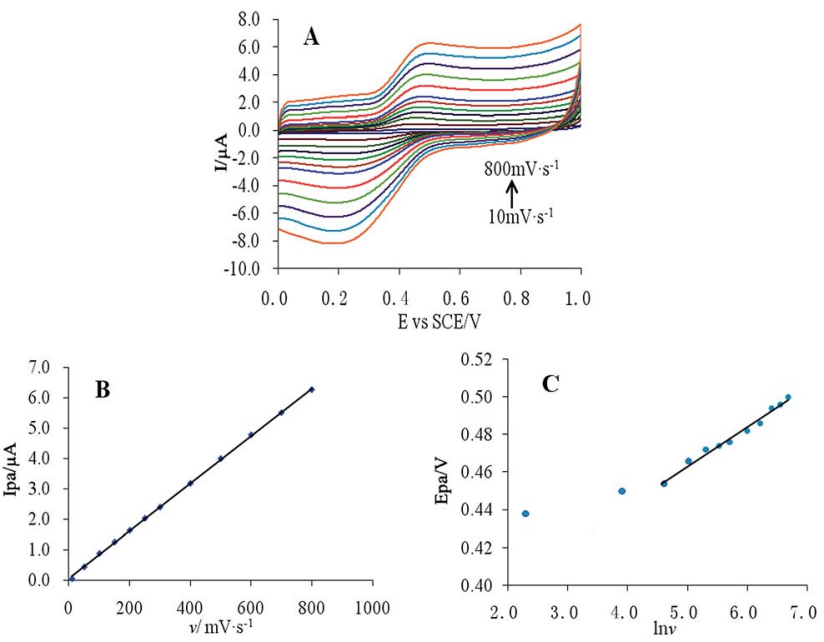

Fig. 4 (A) Cyclic voltammetry of $10 \mu \mathrm{M}$ CUR at different scanning rates, (B) linear relationship of $I_{\mathrm{pa}}$ and $v$, and (C) linear relationship of $I_{\mathrm{pa}}$ and $\ln v$.

$$
E=0.021 \ln v+0.357(r=0.9842)
$$

According to the quasi-reversible electrode reaction, the relationship between peak potential and scanning rate can be described as follows: ${ }^{28}$

$$
E_{\mathrm{pa}}=E^{\theta}-\frac{R T}{\alpha n F}\left[0.78-\ln \frac{k^{0}}{D^{1 / 2}}+\ln \left(\frac{\alpha n F v}{R T}\right)^{1 / 2}\right]
$$

Combining eqn (1) and (2),

$$
\frac{1}{2} \times \frac{R T}{\alpha n F}=0.021
$$

where $\alpha$ is the charge transfer coefficient, $n$ is the number of transferred electrons, $F$ is the Faraday constant (96 $487 \mathrm{C}$ $\left.\mathrm{M}^{-1}\right), v$ is the scanning rate $\left(\mathrm{V} \mathrm{s}^{-1}\right), R$ is the gas constant (8.314 $\mathrm{J} \mathrm{K}^{-1}$ ), and $T$ is the temperature in $\mathrm{K}(298)$. The value of $\alpha n$ was calculated to be 0.61 , assuming $\alpha$ was 0.5 (ref. 29) and the number of electrons involved in the reaction was $n=$ 1.22 , which was close to 1 . In combination with the conclusion of Section 3.2.3, the redox reaction of CUR on this electrode involved transferring one proton and one electron. A reaction mechanism was proposed, as described below (Scheme 2).

In addition, combining eqn (3) and the linear relation between $I_{\text {pa }}$ and $v$ (Fig. 4B), the absorption capacity of CUR on the MIP-CPE surface was calculated as follows: ${ }^{30}$

$$
I_{\mathrm{pa}}=\frac{n^{2} F^{2} \Gamma A v}{4 R T}
$$

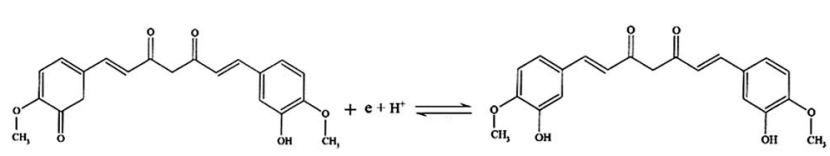

Scheme 2 Redox reaction mechanism of CUR on MIP-CPE. where $\Gamma$ is the absorption capacity $\left(\mathrm{M} \mathrm{cm}^{2}\right), A$ is the electrode surface area $\left(\mathrm{cm}^{2}\right)$, and $n, F, v, R$, and $T$ are as described in eqn (2). The absorption capacity of CUR on the MIP-CPE surface was calculated to be $\Gamma=26.8 \mathrm{nM} \mathrm{cm}^{2}$.

\subsection{Interference test}

For the interference test, 1000 -fold $\mathrm{Mg}^{2+}, \mathrm{Zn}^{2+}, \mathrm{K}^{+}, \mathrm{Na}^{+}, \mathrm{Cl}^{-}$, and $\mathrm{CO}_{3}{ }^{2-}$ were added to a $10 \mu \mathrm{M}$ CUR standard solution and scanned using the CPE and MIP-CPE, respectively. The results showed no interference in the detection of CUR and that the MIP-CPE exhibited a higher anti-interference ability than the bare CPE.

\subsection{Electrode precision, repeatability, and stability}

After obtaining a stable scan for the blank solution, the freshly prepared modified electrode was evaluated by continuously measuring the CUR standard solution ten times. As the relative standard deviation (RSD) was $0.4 \%$, the modified electrode was very precise. Five electrodes were prepared under the same conditions simultaneously. The RSD was $3.3 \%$ when measuring the same CUR standard solution, which indicated good repeatability. After storing the same electrode at room temperature for $3,6,9,12$, and 15 days, the RSD was $2.8 \%$ when measuring the same CUR standard solution, which indicated good stability.

\subsection{Linear range and detection limit}

The peak current was found to be linearly dependent on CUR concentration in the range 0.1-50 $\mu \mathrm{M}$ (Fig. 5). The regression equation was $I_{\mathrm{pa}}=0.065 c+0.322, r=0.9999$. According to $\mathrm{S} / \mathrm{N}=3$, the detection limit was calculated to be $10.1 \mathrm{nM}$.

\subsection{Sample analysis}

The contents of CUR in curcuma powder and cookies were determined using the MIP-CPE. The samples were treated as described in Section 2.5, and the results are summarized in Table 1. Converting to a mass fraction, the contents of CUR in curcuma powder and cookies were $2.10 \%$ and $0.0199 \%$, respectively.

\subsection{Method comparison}

Compared with the reference method (Table 2), the present method had a wider linear range, higher recovery rate, and
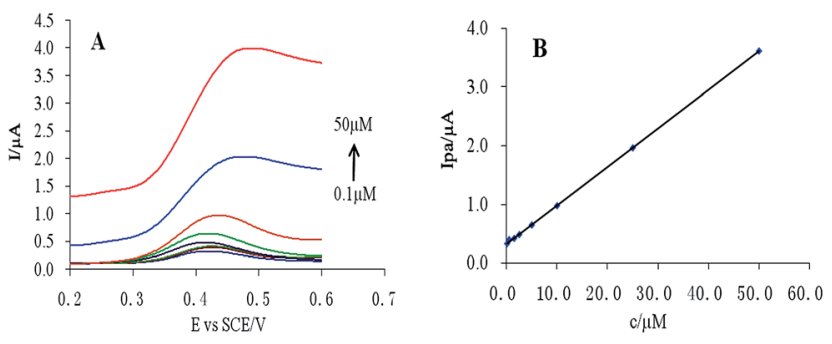

Fig. 5 (A) Linear scan cyclic voltammogram of CUR at different concentrations, and (B) linear relationship between CUR concentration and peak current. 
Table 1 Application of MIP-CPE to the detection of CUR in curcuma powder and cookies $(n=5)$

\begin{tabular}{|c|c|c|c|c|c|}
\hline Samples & Found $/ \mu \mathrm{M}$ & Added $/ \mu \mathrm{M}$ & Determined $/ \mu \mathrm{M}$ & $\begin{array}{l}\text { Recovery, } \\
n=5 / \%\end{array}$ & $\begin{array}{l}\text { RSD, } \\
n=5 / \%\end{array}$ \\
\hline \multirow[t]{3}{*}{ Curcuma powder } & 11.40 & 9.00 & 20.12 & 96.67 & 2.33 \\
\hline & 11.40 & 11.50 & 23.51 & 105.2 & 1.95 \\
\hline & 11.40 & 14.00 & 26.26 & 105.7 & 1.97 \\
\hline \multirow[t]{3}{*}{ Curcuma cookies } & 0.54 & 0.42 & 0.93 & 92.86 & 3.23 \\
\hline & 0.54 & 0.55 & 1.04 & 90.91 & 3.48 \\
\hline & 0.54 & 0.65 & 1.13 & 90.77 & 2.99 \\
\hline
\end{tabular}

Table 2 Comparison of this method with references for CUR detection

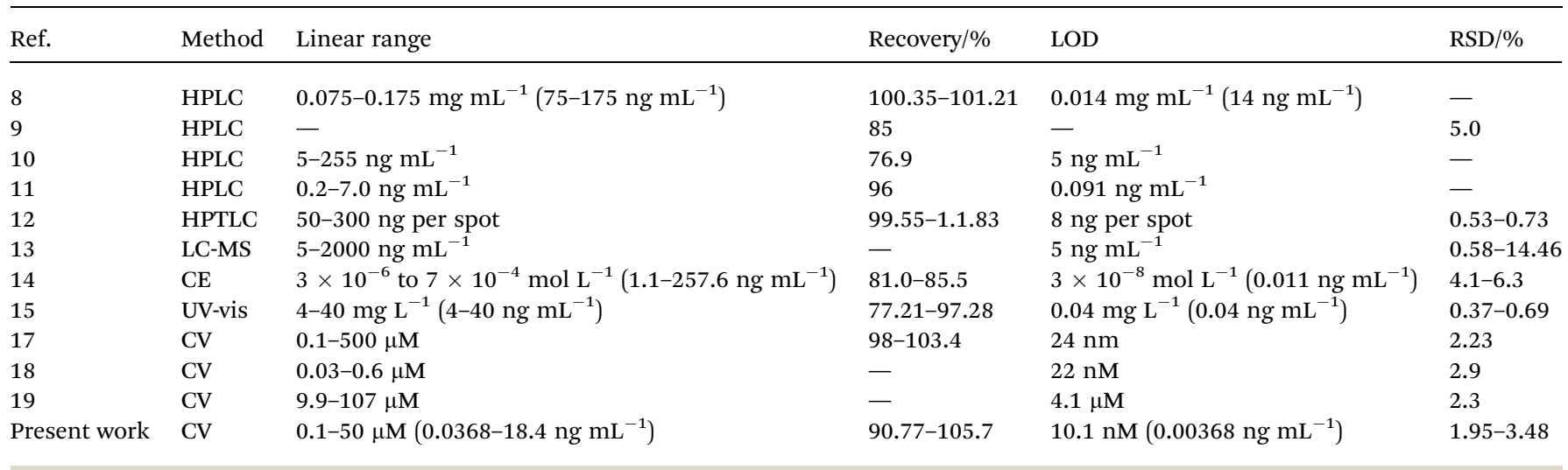

lower detection limit. A small portable electrochemical workstation was used in this study, for which the instrument cost was relatively low and no complex pre-treatment of the sample was needed. Therefore, it could be applied to the on-site rapid detection of CUR in food.

\section{Conclusions}

A CUR-MIP-modified MIP-CPE has been developed. The optimum amount of polymer in the electrode was $20 \%$ and the oxidation peak current at $0.434 \mathrm{~V}$ was approximately 4.5 times higher than that of the bare CPE. The optimum conditions for CUR determination by the electrode were found to be a PBS buffer solution ( $\mathrm{pH}$ 3.06), an accumulation time of $20 \mathrm{~s}$, and a scanning rate of $50 \mathrm{mV} \mathrm{s}^{-1}$. The linear range was $0.1-50 \mu \mathrm{M}$, and the detection limit was $10.1 \mathrm{nM}$. The method was used to determine the CUR content in commercial samples A and B, and the recovery rate ranged from $90.77 \%$ to $105.7 \%$. The electrode showed good sensitivity, selectivity, and antiinterference ability. Its simple sample pretreatment, fast detection speed, and low cost make its application to the rapid determination of CUR in food feasible.

\section{Acknowledgements}

This work was supported by the National Natural Science Foundation of China (No. 81573678) and the Natural Science Foundation of Guangdong province (No. 2015A030313584).

\section{References}

1 Chinese pharmacopoeia, 2015, pp. 264-265.

2 A. B. Hegge, T. T. Nielsen, K. L. Larsen, E. Bruzell and H. H. Tønnesen, J. Pharm. Sci., 2012, 101, 1524-1537.

3 J. Miquel, A. Bernd, J. M. Sempere, J. Díaz Alperi and A. Ramírez, Arch. Gerontol. Geriatr., 2002, 34, 37-46.

4 L. L. Miguel, Mol. Nutr. Food Res., 2008, 52, S103-S127.

5 Y. Guang, G. Kimmer and L. J. Susan, Mol. Carcinog., 2013, 52, 404-411.

6 P. Neerati, R. Devde and A. K. Gangi, Phytother. Res., 2014, 28, 1796-1800.

7 Y. M. Sun, R. X. Wang, S. L. Yuan, X. J. Lin and C. B. Liu, Chin. J. Chem., 2004, 22, 827-830.

8 M. A. Korany, R. S. Haggag, M. A. A. Ragab and O. A. Elmallah, Arabian J. Chem., 2013, DOI: 10.1016/j.arabjc.2013.06.021.

9 M. J. Scotter, LWT-Food Sci. Technol., 2009, 42, 1345-1351.

10 Y. Pak, R. Patek and M. Mayersohn, J. Chromatogr. B: Anal. Technol. Biomed. Life Sci., 2003, 796, 339-346.

11 D. D. Heath, M. A. Pruitt, D. E. Brenner and C. L. Rock, J. Chromatogr. B: Anal. Technol. Biomed. Life Sci., 2003, 783, 287-295.

12 M. J. Ansari, S. Ahmad, K. Kohli, J. Ali and R. K. Khar, J. Pharm. Biomed. Anal., 2005, 39, 132-138.

13 W. Z. Ma, J. L. Wang, Q. Guo and P. F. Tu, J. Pharm. Biomed. Anal., 2015, 111, 215-221.

14 X. H. Sun, C. L. Gao, W. D. Cao, X. R. Yang and E. K. Wang, J. Chromatogr. A, 2002, 962, 117-125. 
15 S. B. R. Aparecida, C. F. Ana, B. Evandro, C. F. Lúcio, H. H. V. F. V. L. Pedro and H. G. Odinei, Food Chem., 2015, 172, 99-104.

16 J. J. B. Nevado, C. G. Cabanillas and A. M. C. Salcedo, Talanta, 1994, 41, 789-797.

17 M. Afzalia, A. Mostafavia and T. Shamspura, Mater. Sci. Eng., C, 2016, 68, 789-797.

18 S. Cakir, E. Bicer and E. Y. Arslan, Croat. Chem. Acta, 2015, 88, 105-112.

19 G. K. Ziyatdinova, A. M. Nizamova and H. C. Budnikov, J. Anal. Chem., 2012, 67, 591-594.

20 C. H. Hu, J. Deng, X. L. Xiao, X. Z. Zhan, K. H. Huang, N. Xiao and S. Q. Ju, Electrochim. Acta, 2015, 158, 298-305.

21 M. Arvand and P. Fallahi, Sens. Actuators, B, 2013, 188, 797805.

22 S. Sadeghi, A. Motaharian and A. Z. Moghaddam, Sens. Actuators, B, 2012, 168, 336-344.
23 Z. P. Liu, H. Y. Zhai, Z. G. Chen, Q. Zhou, Z. X. Liang and Z. H. Su, Electrochim. Acta, 2014, 136, 370-376.

24 H. Y. Zhai, Z. X. Liang, Z. G. Chen, H. H. Wang, Z. P. Liu, Z. H. Su and Q. Zhou, Electrochim. Acta, 2015, 171, 105-113.

25 Z. X. Liang, H. Y. Zhai, Z. G. Chen, H. H. Wang, S. M. Wang, Q. Zhou and X. T. Huang, Sens. Actuators, B, 2016, 24, 915925.

26 X. Y. Liu, L. J. Zhu, X. Gao, Y. X. Wang, H. X. Lu, Y. W. Tang and J. R. Li, Food Chem., 2016, 202, 309-315.

27 P. Wang, W. M. Hu and W. K. Su, Anal. Chim. Acta, 2008, 615, 54-62.

28 J. Wang, Analytical electrochemistry, 2006, p. 30.

29 H. Y. Ma, N. F. Hu and J. F. Rusling, Langmuir, 2000, 16, 4969-4975.

30 J. Wang, Analytical electrochemistry, 2006, p. 34. 